\title{
A characterization of nonemptiness and boundedness of the solution set for set-valued vector equilibrium problems via scalarization and stability results
}

\author{
Pakkapon Preechasilp ${ }^{\text {* }^{*} \text { and Rabian Wangkeeree }}{ }^{2}$
}

${ }^{*}$ Correspondence:

preechasilpp@gmail.com

${ }^{1}$ Program in Mathematics,

Faculty of Education,

Pibulsongkram Rajabhat

University, Phitsanulok 65000,

Thailand

Full list of author information

is available at the end of the

article

\begin{abstract}
In this paper, the existence theorems of solutions for generalized weak vector equilibrium problems are developed in real reflexive Banach spaces. Based on recession method and scalarization technique, we derive a characterization of nonemptiness and boundedness of solution set for generalized weak vector equilibrium problems. Moreover, Painlevé-Kuratowski upper convergence of solution set is also discussed as an application, when both the objective mapping and the constraint set are perturbed by difference parameters.
\end{abstract}

Keywords: Equilibrium problem, Barrier cone, Pseudomonotone mappings, Stability analysis

\section{Background}

Let $X$ be a real reflexive Banach space and $Y$ be a real normed linear space. Suppose that, and $C \subseteq Y$ is a nonempty, closed and convex pointed cone with int $C \neq \varnothing$. Let $K \subseteq X$ be a non-empty subset and a set-valued function $F: K \times K \rightarrow 2^{Y} \backslash\{\varnothing\}$, the following generalized weak vector equilibrium problem (GWVEP) is to find $\bar{x} \in K$ such that

$$
\bar{x} \in K \text { such that } F(\bar{x}, y) \cap(-i n t C)=\varnothing, \forall y \in K \text {, }
$$

(GWVEP)

and the dual problem for (GWVEP), is so called (DGWVEP), is to find $\bar{x} \in K$ such that

$$
\bar{x} \in K \text { such that } F(y, \bar{x}) \cap(\text { int } C)=\varnothing, \forall y \in K \text {. }
$$

(DGWVEP)

Both (GWVEP) and (DGWVEP) have been extensively studied by many authors (see Ansari and Flores-Bazán 2006; Ansari et al. 2001a, b, 2002; Flores-Bazán and FloresBazán 2003; Ansari et al. 2001; Sadeqi and Alizadeh 2011; Zhong et al. 2011). An important and interesting topic for (GWVEP) and (DGWVEP) is to study the nonemptiness and boundedness of the solution sets. As far as we known, the first paper which discussed this issues was Flores-Bazán and Flores-Bazán (2003) in the case where $F$ is vector-valued. They studied the existence of solutions of (GWVEP) under the asymptotic analysis, where neither compactness of $K$ nor any coercivity condition is assumed in

(c) 2016 The Author(s). This article is distributed under the terms of the Creative Commons Attribution 4.0 International License (http://creativecommons.org/licenses/by/4.0/), which permits unrestricted use, distribution, and reproduction in any medium, provided you give appropriate credit to the original author(s) and the source, provide a link to the Creative Commons license, and indicate if changes were made. 
reflexive Banach spaces. By using idea of recession method in Flores-Bazán and FloresBazán (2003), Ansari and Flores-Bazán (2006) gave some necessary and sufficient conditions for nonemptiness and boundedness of the solution set of (GWVEP). In 2011, Sadeqi and Alizadeh (2011) discussed and improved some results of Ansari and FloresBazán (2006). They gave the conditions under which the solution set of (GWVEP) is non-empty, convex and weakly compact subset in reflexive Banach spaces. After a thorough review of the literature and according to our knowledge, we found that the convexity assumed for second variable of $F$ is an essential assumption (see also Chen et al. 2008; Flores-Bazán 2000; Fang and Huang 2007).

On the other hand, the stability analysis of the solution mappings to generalized vector equilibrium problem is an important topic in vector optimization theory. Recently, the lower semicontinuity, (Hölder) continuity of the solution maps to (GWVEP) are discussed in Li and Li (2011), Gong (2008), Chen et al. (2009), Xu and Li (2013). Among those papers, we observe that the linear scalarization technique is one effective to deal with the lower semicontinuity and (Hölder) continuity of solution mappings to (GWVEP). Based on the linear scalarization, the solution sets for (GWVEP) is the union of family of the solution set to scalarized equilibrium problems with respect to the linear map on dual cone. In natural, the union of family of solution sets to scalarized equilibrium problems is finer than the solution set to (GWVEP). In order to obtain the equality, convexity in second variable of $F$ is assumed.

Motivated and Inspired by above works, the aim of this paper is to consider a (GWVEP) with a set-valued map on unbounded constraint set in reflexive Banach spaces. We first collect the characterization results of the nonemptiness and boundedness of the solution set of (GWVEP). By using the linear scalarization technique, we characterize the nonemptiness and boundedness of the solution set of (GWVEP) in terms of nonemptiness and boundedness of a family of scalar equilibrium problem with respect to linear maps in connected base for dual cone of $C$. Finally, we give the stability results for the solution maps to (GWVEP) in the sense of Painlevé-Kuratowski upper convergence of solution set.

The paper is organized as follows. In "Preliminaries" section, we introduce some basic notations and preliminary results. In "Characterization of nonemptiness and boundedness of the solution set" section, by using a scalarization technique, we establish the nonemptiness and boundedness of solution set for (GWVEP) in reflexive Banach spaces. In "Stability analysis" section, we give an application to the stability of the solution sets for (GWVEP).

\section{Preliminaries}

Throughout this paper, unless otherwise specified, we always assume that $X$ is a real reflexive Banach space, $Y$ is a real normed space with dual space $Y^{*}$ and $C \subseteq Y$ is a nonempty, closed, convex and pointed cone with int $C \neq \varnothing$. Let

$$
C^{*}:=\left\{\xi \in Y^{*}:\langle\xi, y\rangle \geq 0, \quad \forall y \in C\right\}
$$

be the dual cone of $C$. Clearly,

$$
\begin{aligned}
y \in C & \Leftrightarrow\langle\xi, y\rangle \geq 0, \quad \forall \xi \in C^{*}, \\
y \in \text { int } C & \Leftrightarrow\langle\xi, y\rangle>0, \quad \forall \xi \in C^{*} .
\end{aligned}
$$


Since int $C \neq \varnothing$, for any fixed $e \in \operatorname{int} C$, it proved in Huang et al. (2014) that the dual cone $C^{*}$ of $C$ has a following weak * compact base $C^{* 0}$.

$$
C^{* 0}:=\left\{\xi \in C^{*}:\langle\xi, e\rangle=1\right\},
$$

where a subset $D \subset C^{*}$ is said to be a base of $C^{*} \Leftrightarrow 0 \notin D$ and $C^{*} \subset \cup_{t \geq 0} t D$.

A vector $x \in K$ is called weak efficient solution to the (GWVEP) if

$$
F(x, y) \cap(- \text { int } C)=\varnothing, \quad \forall y \in K,
$$

and weak efficient solution to the (DGWVEP) if

$$
F(y, x) \cap(\text { int } C)=\varnothing, \quad \forall y \in K .
$$

Denote by $S_{W}^{P}(K, F)$ and $S_{W}^{D}(K, F)$ the set of all weak efficient solution to the (GWVEP) and (DGWVEP), respectively.

Definition 1 (Zhong et al. 2011) Let $K$ be a non-empty convex subset of $X$. For a given closed convex cone $C$ of a real normed space $Y$, the set-valued map $F: K \rightarrow 2^{Y} \backslash\{\varnothing\}$ is said to be

(i) upper $C$-convex, if for any $x, y \in K$ and for any $t \in[0,1]$,

$$
t F(x)+(1-t) F(y) \subseteq F(t x+(1-t) y)+C ;
$$

(ii) lower $C$-convex, if for any $x, y \in K$ and for any $t \in[0,1]$,

$$
F(t x+(1-t) y) \subseteq t F(x)+(1-t) F(y)-C ;
$$

(iii) $C$-convex, if $F$ is both upper $C$-convex and lower $C$-convex.

Remark 1 If $F$ is a upper $C$-convex map on $K$, then for any $x \in K, F(x)+C$ is convex set.

We first recall the well-known concept of monotone mapping for a real set-valued mapping.

Definition 2 A bifunction $f: K \times K \rightarrow 2^{\mathbb{R}} \backslash\{\varnothing\}$ is said to be

(i) monotone on $K$, if for any $x, y \in K$

$$
z+z^{\prime} \leq 0, \quad \forall z \in f(x, y), z^{\prime} \in f(y, x) ;
$$

(ii) pseudomonotone on $K$, if for any $x, y \in K$

$$
z \geq 0, \quad \forall z \in f(x, y) \Rightarrow z^{\prime} \leq 0, \forall z^{\prime} \in f(y, x) .
$$

It is well-known that every monotone map is pseudomonotone map.

In the case where $F$ is a vector set-valued, the concept of monotonicity can be also extended as follows.

Definition 3 Let $C \subseteq Y$ be a nonempty, closed, convex and pointed cone with int $C \neq \varnothing$. A set-valued map $F: K \times K \rightarrow 2^{Y} \backslash\{\varnothing\}$ is said to be 
(i) $C$-monotone if, for all $x, y \in K$,

$$
F(x, y)+F(y, x) \subseteq-C ;
$$

(ii) $C$-pseudomonotone type $I$ if, for all $x, y \in K$,

$$
F(x, y) \cap(-\operatorname{int} C)=\varnothing \Rightarrow F(y, x) \cap(\text { int } C)=\varnothing ;
$$

(iii) $C$-pseudomonotone type $I I$ if, for all $x, y \in K$,

$$
F(x, y) \cap(- \text { int } C)=\varnothing \Rightarrow F(y, x) \subseteq-C ;
$$

(iv) $\xi$-monotone w.r.t. $C^{*}$ if, for any $\xi \in C^{*}$ and for any $x, y \in K$,

$$
\xi(z)+\xi\left(z^{\prime}\right) \leq 0, \quad \forall z \in F(x, y), \quad \forall z^{\prime} \in F(y, x) ;
$$

(v) $\xi$-pseudomonotone w.r.t. $C^{*}$ if, for any $\xi \in C^{*}$ and for any $x, y \in K$,

$$
\xi(z) \geq 0, \quad \forall z \in F(x, y) \Rightarrow \xi\left(z^{\prime}\right) \leq 0, \quad \forall z^{\prime} \in F(y, x) .
$$

Remark 2 (1) It is clear that $C$-monotone mapping is $C$-pseudomonotone type $I$ and type $I I$ and $C$-pseudomonotone type $I I$ implies $C$-pseudomonotone type $I$.

(2) Every $C$-monotone mapping is $\xi$-pseudomonotone w.r.t. $C^{*}$.

(3) Every $C$-pseudomonotone type $I I$ mapping is $\xi$-pseudomonotone w.r.t. $C^{*}$, Indeed, for any $\xi \in C^{*}$ and for any $x, y \in K$ satisfying $\xi(z) \geq 0$ for all $z \in F(x, y)$, we have $z \notin-$ int $C$ and so $F(x, y) \cap(-$ int $C)=\varnothing . F(y, x) \subseteq-C$ implies that $\xi\left(z^{\prime}\right) \leq 0$ for all $z^{\prime} \in F(y, x)$. But, $C$-pseudomonotone type $I$ may not implies $\xi$-pseudomonotone w.r.t. $C^{*}$.

Example 1 Let $X=\mathbb{R}, K=[0,1], Y=\mathbb{R}^{2}, C=\mathbb{R}_{+}^{2}$. Define $F: K \times K \rightarrow 2^{Y} \backslash\{\varnothing\}$ by

$$
F(x, y)= \begin{cases}(x,-x) & \text { if } x=y, \\ \{(y-x)\} \times[0,(y-x)] & \text { if } y-x>0, \\ \{(y-x)\} \times[(y-x), 0] & \text { if } y-x<0 .\end{cases}
$$

Thus, clearly that $F$ is $\xi$-pseudomonotone on $K$ w.r.t. $C^{*} \equiv C$. Indeed, for any $x, y \in K$ and $\xi \in C^{*}$ if $\xi(F(x, y)) \geq 0$, then $y-x>0$. This implies that

$$
F(y, x)=\{(x-y)\} \times[(x-y), 0] \subseteq-\mathbb{R}_{+}^{2} \Rightarrow \xi(z) \leq 0, \quad \forall z \in F(y, x) .
$$

But $C$-pseudomonotone type $I I$ in the case when $x=y$.

Example 2 Let $X=\mathbb{R}, K=[0,+\infty), Y=\mathbb{R}^{2}, C=\mathbb{R}_{+}^{2} \quad$ and $\quad C^{*} \equiv C . \quad$ Define $F: K \times K \rightarrow 2^{Y} \backslash\{\varnothing\}$ by

$$
F(x, y)=\{0\} \times[0,|y-x|], \quad \forall x, y \in K .
$$

Thus, clearly that for any $x, y \in K, F(x, y) \cap(-$ int $C)=\varnothing$ implies that $F(y, x) \cap($ int $C)=\varnothing$. Hence, $F$ is pseudomonotne on $K$ type $I$, but not $C$-pseudomonotone type $I I$.

Moreover, for any $\xi \in C^{*}$ and $x, y \in K$, we then have

$$
\xi(F(x, y))=\xi(F(y, x)) \geq 0 .
$$


Therefore, $F$ is not $\xi$-pseudomonotone on $K$ w.r.t. $C^{*}$ as shown in the following example.

Definition 4 A topological space $E$ is said to be connected iff, it is not the union of two disjoint nonempty open sets. Moreover, $E$ is said to be path-connected iff, any two points of $E$ can be joined by a path.

The following lemma, which gives an equivalent characterization of connected spaces, plays an important role in our proof.

Lemma 1 A topological space $E$ is connected if and only if the only subsets of $E$ which are both open and closed are $E$ and $\varnothing$.

Definition 5 Let $F: K \rightarrow 2^{Y}$ be a set-valued mapping with nonempty values. $F$ is said to be

(i) upper semicontinuous(u.s.c.) on $K$ iff, for every $x \in K$ and every neighborhood $N(F(x))$ of $F(x)$, there exists a neighborhood $N(x)$ of $x$ such that $F(N(x)) \subseteq N(F(x)$ );

(ii) lower semicontinuous(l.s.c.) on $K$ iff, for every $x \in K, u \in F(x)$ and every neighbor$\operatorname{hood} N(u)$ of $u$, there exists a neighborhood $N(x)$ of $x$ such that $F\left(x^{\prime}\right) \cap N(u) \neq \varnothing$ for every $x^{\prime} \in N(x)$.

Proposition 1 (Aubin and Ekeland 1984; Ferro 1989)

(i) F is l.s.c. at $\bar{\lambda}$ if and only if for any sequence $\left\{\lambda_{n}\right\} \subset \Lambda$ with $\lambda_{n} \rightarrow \bar{\lambda}$ and any $\bar{x} \in F(\bar{\lambda})$, there exists $x_{n} \in F\left(\lambda_{n}\right)$ such that $x_{n} \rightarrow \bar{x}$.

(ii) $F$ is weakly l.s.c. at $\bar{\lambda}$ if and only if for any sequence $\left\{\lambda_{n}\right\} \subset \Lambda$ with $\lambda_{n} \rightarrow \bar{\lambda}$ and any $\bar{x} \in F(\bar{\lambda})$, there exists $x_{n} \in F\left(\lambda_{n}\right)$ such that $x_{n} \rightarrow \bar{x}$.

(iii) If $F$ has compact values (i.e., $F(\lambda)$ is a compact set for each $\lambda \in \Lambda$ ), then $F$ is u.s.c. at $\bar{\lambda}$ if and only if for any sequence $\left\{\lambda_{n}\right\} \subset \Lambda$ with $\lambda_{n} \rightarrow \bar{\lambda}$ and for any $x_{n} \in F\left(\lambda_{n}\right)$, there exists $\bar{x} \in F(\bar{\lambda})$ and a subsequence $\left\{x_{n_{k}}\right\}$ of $\left\{x_{n}\right\}$ such that $x_{n_{k}} \rightarrow \bar{x}$.

We collect the following well-known KKM-Fan lemma.

Lemma 2 (Fan 1984) Let $M$ be a nonempty, closed and convex subset of $X$ and $F: M \rightarrow 2^{M} \backslash\{\varnothing\}$ be a set-valued map. Suppose that for any finite set $\left\{x_{1}, \ldots, x_{m}\right\} \subseteq M$, one has

(i) $\operatorname{conv}\left\{x_{1}, \ldots, x_{m}\right\} \subset \cup_{i=1}^{m} F\left(x_{i}\right)$ (i.e., $F$ is a KKM map on $M$ );

(ii) $F(x)$ is closed for every $x \in M$; and

(iii) $F(x)$ compact for some $x \in M$.

Then $\cap_{x \in M} F(x) \neq \varnothing$.

Now, we recall the fundamental tools used throughout this paper. This leads to the concepts of asymptotic cone and asymptotic function through its epigraph.

$$
K_{\infty}=\left\{d \in X: \exists t_{k} \rightarrow+\infty, x_{k} \in X \text { such that } \frac{1}{t_{k}} x_{k} \rightarrow d\right\},
$$


where " $\rightarrow$ " or " $\omega-\lim _{n \rightarrow \infty} x_{n}=x$ " means convergence in the weak topology. In case $K$ is convex subset, $K_{\infty}$ can also be determined by the following formula

$$
K_{\infty}=\left\{d \in X: x_{0}+t d \in K, \forall t>0, \quad \forall x_{0} \in K\right\} .
$$

The barrier cone of $K$ is defined by

$$
\operatorname{barr} K=\left\{\xi^{*} \in K^{*}: \sup _{y \in K}\left\langle\xi^{*}, y\right\rangle<+\infty\right\} \text {. }
$$

Proposition 2 (Ansari and Flores-Bazán 2006, Proposition 2.1) The following holds:

(i) $K^{1} \subseteq K^{2}$ implies $K_{\infty}^{1} \subseteq K_{\infty}^{2}$;

(ii) $(K+x)_{\infty}=K_{\infty}, \forall x \in X$;

(iii) let $\left\{K^{i}\right\}_{i \in I}$ be any family of nonempty sets in $X$, then

$$
\left(\cap_{i \in I} K^{i}\right)_{\infty} \subset \cap_{i \in I} K_{\infty}^{i}
$$

If, in addition, $\cap_{i \in I} K^{i} \neq \varnothing$ and each set $K^{i}$ is closed and convex, then we obtain an equality in (3).

Lemma 3 (Adly et al. 2004) Let $K$ be a nonempty, closed and convex subset of a real reflexive Banach space $X$ with int $($ barr $K) \neq \varnothing$. Then there is no sequence $\left\{x_{n}\right\} \subset K$ with $\left\|x_{n}\right\| \rightarrow \infty$ such that origin is a weak limit of $\frac{x_{n}}{\left\|x_{n}\right\|}$, i.e. $\frac{x_{n}}{\left\|x_{n}\right\|} \rightarrow 0$.

Lemma 4 (Fan and Zhong 2008) Let $K$ be a nonempty, closed, convex subset of a real reflexive Banach space $X$ with int $($ barr $(K)) \neq \varnothing$. Then there exists no sequence $\left\{d_{n}\right\} \subset K_{\infty}$ with each $\left\|d_{n}\right\|=1$ such that $d_{n} \rightarrow 0$.

Lemma 5 (Fan and Zhong 2008) Let $(M, d)$ be a metric space and $\mu_{0} \in M$ be a given point. Let $K: M \rightarrow 2^{X}$ be a set-valued mapping with nonempty valued and upper semicontinuous at $\mu_{0}$. Then there exists a neighborhood $N\left(\mu_{0}\right)$ of $\mu_{0}$ such that $\left(K(\mu)_{\infty}\right) \subset\left(K\left(\mu_{0}\right)\right)_{\infty}$ for all $\mu \in N\left(\mu_{0}\right)$.

\section{Characterization of nonemptiness and boundedness of the solution set}

In this section, we shall prove the characterization of nonemptiness and boundedness of the solution set for (GWVEP) which states that under suitable conditions.

First of all, we recall the existing assumptions and results which can be found in Ansari and Flores-Bazán (2006), Zhong et al. (2011), Sadeqi and Alizadeh (2011).

Assumption 1 (Zhong et al. 2011; Ansari and Flores-Bazán 2006) The set-valued map $F: K \times K \rightarrow 2^{Y} \backslash\{\varnothing\}$ is such that:

$\left(F_{0}\right) \quad F(x, x)=\{0\}$ for all $x \in K$.

$\left(F_{1}\right)$ For any $x, y \in K, F(x, y) \cap(-$ int $C)=\varnothing \Rightarrow F(y, x) \subseteq-C$ (C pseudomonotone type II). 
$\left(F_{2}\right)$ For any $x \in K, F(x, \cdot): K \rightarrow 2^{Y} \backslash\{\varnothing\}$ is $C$-convex.

$\left(F_{3}\right) \quad$ For any $x, y \in K$, the set $\{z \in[x, y]: F(z, y) \cap(-\operatorname{int} C)=\varnothing\}$ is closed, where $[x, y]$ denotes the closed line segment joining $x$ and $y$.

$\left(F_{4}\right)$ For any $x \in K, F(x, \cdot)$ is weakly lower semicontinuous.

$\left(F_{5}\right)$ For any $y \in K,\{x \in K: F(y, x) \cap($ int $C)=\varnothing\}$ is convex.

Under Assumption 1, It is proved in Zhong et al. (2011) that, $S_{W}^{P}(K, F)$ is nonempty if $K$ is bounded subset of $X$. In the case where $K$ is unbounded, it is needed to determine the behavior of $F$ along some particular directions. We introduce the following cones.

$$
R_{1}:=\left\{d \in K_{\infty}: F(y, y+t d) \cap(\operatorname{int} C)=\varnothing, \quad \forall y \in K, t>0\right\} .
$$

The following lemma illustrates that the solution set $S_{W}^{P}(K, F)$ and $S_{W}^{D}(K, F)$ are coincide no matter what $K$ is bounded or not.

Lemma 6 (Sadeqi and Alizadeh 2011, Lemma 3.4) Let $K$ be a nonempty, closed and convex subset of $X$ and $F: K \times K \rightarrow 2^{Y} \backslash\{\varnothing\}$ be a set valued map satisfying $\left(F_{0}\right)-\left(F_{3}\right)$. Then

$$
S_{W}^{P}(K, F)=S_{W}^{D}(K, F) .
$$

Theorem 1 (Sadeqi and Alizadeh 2011, Theorem 3.5) Let $K$ be a nonempty, closed and convex subset of $X$ and $F: K \times K \rightarrow 2^{Y} \backslash\{\varnothing\}$ be a set valued map satisfying $\left(F_{0}\right)-\left(F_{5}\right)$. If the set the solution set $S_{W}^{P}(K, F)$ is nonempty, then

$$
\left(S_{W}^{P}(K, F)\right)_{\infty}=\left(S_{W}^{D}(K, F)\right)_{\infty}=R_{1} .
$$

The following theorem is due to the result in Zhong et al. (2011), Ansari and FloresBazán (2006), Sadeqi and Alizadeh (2011).

Theorem 2 Let $K$ be a nonempty closed convex subset of $X$ and $F: K \times K \rightarrow 2^{Y} \backslash\{\varnothing\}$ be a set valued mapping satisfying assumptions $\left(F_{0}\right)-\left(F_{5}\right)$. Suppose that $\operatorname{int}(\operatorname{barr}(K)) \neq \varnothing$. Then the following statements are equivalent.

(i) the solution set of $S_{W}^{P}(K, F)$ is nonempty and bounded;

(ii) the solution set of $S_{W}^{D}(K, F)$ is nonempty and bounded;

(iii) $R_{1}=\{0\}$;

(iv) there exists a bounded set $B \subset K$ such that for every $x \in K \backslash B$, there exists some $y \in B$ such that $F(y, x) \cap($ int $C) \neq \varnothing$.

Proof (i) $\Leftrightarrow$ (ii) and (ii) $\Rightarrow$ (iii) are obtained by Theorems 1 and 2, respectively.

(iii) $\Rightarrow$ (iv) Suppose not, if (iv) does not hold, then there exists a sequence $\left\{x_{n}\right\} \subseteq K$ such that for each $n,\left\|x_{n}\right\|>n$ and

$$
F\left(y, x_{n}\right) \cap(\text { int } C)=\varnothing,
$$

for every $y \in K$ with $\|y\| \leq n$. For fixed $y \in K$ and $t>0$, without loss of generality, we may take a subsequence $\left\{x_{n_{k}}\right\}$ of $\left\{x_{n}\right\}$ such that 


$$
\frac{t}{\left\|x_{n_{k}}-y\right\|} \in(0,1) \quad \text { and } \quad \omega-\lim _{k \rightarrow+\infty} \frac{x_{n_{k}}-y}{\left\|x_{n_{k}}-y\right\|}=\omega-\lim _{k \rightarrow+\infty} \frac{x_{n_{k}}}{\left\|x_{n_{k}}\right\|}=d_{0} \in K_{\infty} .
$$

Thanks to Lemma 3 , one has $d_{0} \neq 0$. The lower $C$-convexity of $F(x, \cdot)$ implies

$$
F\left(y, y+\frac{t\left(x_{n_{k}}-y\right)}{\left\|x_{n_{k}}-y\right\|}\right) \subseteq\left(1-\frac{t}{\left\|x_{n_{k}}-y\right\|}\right) F(y, y)+\frac{t}{\left\|x_{n_{k}}-y\right\|} F\left(y, x_{n_{k}}\right)-C .
$$

It follows from $F(y, y)=\{0\}$ and $F\left(y, x_{n_{k}}\right) \cap($ int $C)=\varnothing$ that

$$
F\left(y, y+\frac{t\left(x_{n_{k}}-y\right)}{\left\|x_{n_{k}}-y\right\|}\right) \cap(\operatorname{int} C)=\varnothing .
$$

Since $y+\frac{t\left(x_{n_{k}}-y\right)}{\left\|x_{n_{k}}-y\right\|} \rightarrow y+t d_{0}$ and $F$ is weakly lower semicontinuous at second argument, we have that $F\left(y, y+t d_{0}\right) \cap($ int $C)=\varnothing$, and so $d_{0} \in R_{1}$. This is a contradiction. Hence (iv) holds.

(iv) $\Rightarrow$ (ii) Let $G: K \rightarrow 2^{K}$ be a set-valued mapping defined by

$$
G(y):=\{x \in K: F(y, x) \cap(\text { int } C)=\varnothing\}, \quad \forall y \in K .
$$

We first prove that $G(y)$ is a closed subset of $K$. Indeed, for any $x_{n} \in G(y)$ with $x_{n} \rightarrow x_{0}$, we have $F\left(y, x_{n}\right) \cap($ int $C)=\varnothing$. It follows from the weakly lower semicontinuity of $F(x, \cdot)$ that $F\left(y, x_{0}\right) \cap($ int $C)=\varnothing$. This shows that $x_{0} \in G(y)$ and so $G(y)$ is closed.

Next, we will show that $G$ is a KKM mapping. Suppose to the contrary that there exist $\quad \alpha_{1}, \alpha_{2}, \ldots, \alpha_{n} \in(0,1) \quad$ with $\quad \alpha_{1}+\alpha_{2}+\cdots+\alpha_{n}=1, y_{1}, y_{2}, \ldots, y_{n} \in K \quad$ and $\bar{y}=\alpha_{1} y_{1}+\alpha_{2} y_{2}+\cdots+\alpha_{n} y_{n} \in \operatorname{co}\left\{y_{1}, y_{2}, \ldots, y_{n}\right\}$ such that $\bar{y} \notin \cup_{i \in\{1,2, \ldots, n\}} G\left(y_{i}\right)$. Then

$$
F\left(y_{i}, \bar{y}\right) \cap(\text { int } C) \neq \varnothing, \quad i=1,2, \ldots, n .
$$

Using $\left(F_{1}\right)$ yields

$$
F\left(\bar{y}, y_{i}\right) \cap(-\operatorname{int} C) \neq \varnothing, \quad i=1,2, \ldots, n .
$$

The upper $C$-convexity of $F$ implies

$$
\alpha_{1} F\left(\bar{y}, y_{1}\right)+\alpha_{2} F\left(\bar{y}, y_{2}\right)+\cdots+\alpha_{n} F\left(\bar{y}, y_{n}\right) \subseteq F(\bar{y}, \bar{y})+C=0+C \subseteq C .
$$

This is a contradiction with (7). Therefore, $G$ is KKM mapping.

We may assume that $B$ is a bounded closed convex set (otherwise, consider the closed convex hull of $B$ instead of $B$ ). Let $\left\{y_{1}, \ldots, y_{m}\right\}$ be finite number of points in $K$ and let $M:=\operatorname{co}\left(B \cup\left\{y_{1}, y_{2}, \ldots, y_{m}\right\}\right)$. Then the reflexivity of the space $X$ yields that $M$ is weakly compact convex. We consider the set-valued mapping $G^{\prime}$ which defined by $G^{\prime}(y):=G(y) \cap M$ for all $y \in M$. Then each $G^{\prime}(y)$ is a weakly compact convex subset of $M$ and $G^{\prime}$ is a KKM mapping. We claim that

$$
\varnothing \neq \cap_{y \in M} G^{\prime}(y) \subset B .
$$

By Lemma 2, the intersection in (8) is nonempty. Moreover, if there exists some $x_{0} \in \cap_{y \in M} G^{\prime}(y)$ but $x_{0} \notin B$, then by (iv), we have $F\left(y, x_{0}\right) \cap$ (int $\left.C\right) \neq \varnothing$ for some $y \in B$. Thus, $x_{0} \notin G(y)$ and so $x_{0} \notin G^{\prime}(y)$, which is a contradiction to the choice of $x_{0}$. 
Let $z \in \cap_{y \in M} G(y)$. Then, by (8) we get $z \in B$, and so $z \in \cap_{i=1}^{m}\left(G\left(y_{i}\right) \cap B\right)$. This shows that the collection $\{G(y) \cap B: y \in K\}$ has finite intersection property. For each $y \in K$, it follows from the weak compactness of $G(y) \cap B$ that $\cap_{y \in K}(G(y) \cap B)$ is nonempty, which coincides with the solution set of $S_{W}^{D}(F, K)$. The proof is complete.

The following example show that Theorem 2 is applicable.

Example 3 Let $X=\mathbb{R}, K=[0,+\infty), Y=\mathbb{R}^{2}, C=\mathbb{R}_{+}^{2}, e=(1,1) \in$ int $C$. A set-valued $\operatorname{map} F: K \times K \rightarrow 2^{\mathbb{R}^{2}} \backslash\{\varnothing\}$ is defined by

$$
F(x, y)=\{y-x\} \times[(y-x),(1+x)(y-x)], \quad \forall x, y \in K .
$$

We have that $K_{\infty}=[0,+\infty)$ and $C^{* 0}:=\left\{\left(x_{1}, x_{2}\right) \in \mathbb{R}^{2}, x_{1}+x_{2}=1, x_{1} \geq 0\right.$ and $\left.x_{2} \geq 0\right\}$. It is easily seen that $F$ is satisfied conditions $\left(F_{0}\right)-\left(F_{4}\right)$. To verify $\left(F_{5}\right)$ holds, we fixed $\bar{y} \in[0,+\infty)$ and consider the following set,

$$
\begin{aligned}
\{x \in K: F(\bar{y}, x) \cap \text { int } C=\varnothing\} & =\{x \in[0,+\infty): x-\bar{y} \leq 0 \text { or }(1+y)(x-\bar{y}) \leq 0\} \\
& =\{x \in[0,+\infty): x \leq \bar{y}\}=[0, \bar{y}] \text { is convex set. }
\end{aligned}
$$

Obviously,

$$
\begin{aligned}
R_{1} & =\left\{d \in K_{\infty}: F(y, y+t d) \cap(\text { int } C)=\varnothing, \forall y \in K, t>0\right\} \\
& =\{d \in[0,+\infty): t d \leq 0, \forall t>0 \text { and } \forall y \in[0,+\infty)\}=\{0\} .
\end{aligned}
$$

Hence, Theorem 2 concludes that $S_{W}^{P}(F, K)$ is nonempty and bounded. It follows from direct calculating that $S_{W}^{P}(F, K)=\{0\}$.

In what follow, we shall discuss the relationship between the nonemptiness and boundedness of the solution set for (GWVEP) and the solution set for (GWVEP) which $F$ is composed by $\xi \in C^{*}$. We recall the concept of $\xi$-efficient solution set for (GWVEP) as follows.

For any fixed $\xi \in C^{* 0}$, the real set-valued map $\xi(F): K \times K \rightarrow 2^{\mathbb{R}} \backslash\{\varnothing\}$ is defined by

$$
\xi(F)(x, y):=\{\xi(z): z \in F(x, y)\}, \quad \forall x, y \in K .
$$

A vector $x \in K$ is called $\xi$-weak efficient solution to the (GWVEP) if

$$
\inf _{z \in F(x, y)} \xi(z) \geq 0, \quad \forall y \in K
$$

and $\xi$-weak efficient solution to the (DGWVEP) if

$$
\sup _{z \in F(y, x)} \xi(z) \leq 0, \quad \forall y \in K
$$

Denote by $S_{\xi}^{P}(K, F)$ and $S_{\xi}^{D}(K, F)$ the set of all $\xi$-weak efficient solution to the (GWVEP) and (DGWVEP), respectively.

The following lemma characterizes relation between $S_{W}^{P}(K, F)$ and $S_{\xi}^{P}(K, F)$.

Lemma 7 Suppose that int $C \neq \varnothing$ and for any $x \in K, F(x, K)+C$ is a convex set. Then,

$$
S_{W}^{P}(K, F)=\cup_{\xi \in C^{*} \backslash\{0\}} S_{\xi}^{P}(K, F)=\cup_{\xi \in C^{*} 0} S_{\xi}^{P}(K, F) .
$$


Proof (つ) Let $x_{0} \in \cup_{\xi \in C^{* 0}} S_{\xi}^{P}(K, F)$. Then there exists $\xi_{0} \in C^{* 0}$ such that

$$
\xi_{0}(z) \geq 0 \text { for all } y \in K \text { for all } z \in F\left(x_{0}, y\right)
$$

We claim that $x_{0} \in S_{W}^{P}(K, F)$. If not, then there exists $y_{0} \in K$ such that

$$
\xi_{0}\left(z_{0}\right)<0 \quad \text { for some } z_{0} \in F\left(x_{0}, y_{0}\right) \text {. }
$$

This is a contradiction with (10). Hence, we have desired.

$(\subseteq)$ Let $x_{0} \in S_{W}^{P}(K, F)$. Then,

$$
F\left(x_{0}, y\right) \cap(-i n t C)=\varnothing \quad \text { for all } \quad y \in K .
$$

This implies that

$$
F\left(x_{0}, K\right) \cap(-i n t C)=\varnothing .
$$

Since $C$ is a pointed convex cone, we have

$$
\left(F\left(x_{0}, K\right)+C\right) \cap(-i n t C)=\varnothing .
$$

Using the separation theorem for convex sets, there exists some $\xi^{\prime} \in Y^{*} \backslash\{0\}$ such that

$$
\inf \left\{\xi^{\prime}\left(F\left(x_{0}, y\right)+c: y \in K, c \in C\right)\right\} \geq \sup \left\{\xi^{\prime}(-c): c \in C\right\} .
$$

From (11), we get $\xi^{\prime} \in C^{*} \backslash\{0\}$ and so

$$
\xi^{\prime}(z) \geq 0 \text { for all } z \in F\left(x_{0}, y\right) \text { for all } y \in K \text {. }
$$

By our hypothesis, we have $C^{* 0}$ is a weakly compact base for $C^{*}$ and we can choose $e \in$ int $C$ with $\xi^{\prime}(e)>0$. Setting $\xi^{\prime \prime}=\frac{\xi^{\prime}}{\xi^{\prime}(e)}$, we then have that $\xi^{\prime \prime} \in C^{* 0}$ and

$$
\xi^{\prime \prime}(z) \geq 0 \text { for all } z \in F\left(x_{0}, y\right) \text { for all } y \in K \text {. }
$$

Hence, $x_{0} \in S_{\xi^{\prime \prime}}^{P}(K, F) \subseteq \cup_{\xi \in C^{* 0}} S_{\xi}^{P}(K, F)$. This completes the proof.

The following corollary give the sufficient conditions for nonemptiness and boundedness of solution set for (GWVEP) in the case of real set-valued mappings.

It follows from Theorem 2, we can derive the following corollary in the case where $F: K \times K \rightarrow 2^{\mathbb{R}} \backslash\{\varnothing\}$.

Corollary 1 Let $K$ be a nonempty closed convex subset of $X$ and $F: K \times K \rightarrow 2^{\mathbb{R}} \backslash\{\varnothing\}$ be a set-valued mapping satisfying assumptions $\left(F_{0}\right)-\left(F_{4}\right)$. Suppose that int $(\operatorname{barr}(K)) \neq \varnothing$. Then the following statements are equivalent.

(i) the solution set of $S_{W}^{P}(K, F)$ is nonempty and bounded;

(ii) the solution set of $S_{W}^{D}(K, F)$ is nonempty and bounded;

(iii) $R=\left\{d \in K_{\infty}: \sup _{z \in F(y, y+t d)} z \leq 0, \forall y \in K, t>0\right\}=\{0\}$;

(iv) there exists a bounded set $B \subset K$ such that for every $x \in K \backslash B$, there exists $y \in B$ such that $z>0$ for some $z \in F(y, x)$.

Proof We see that $F$ satisfies the assumption $\left(F_{0}\right)-\left(F_{4}\right)$ in Theorem 2 . It is easy to verify, by $\left(F_{2}\right)$, that $\left(F_{5}\right)$ is satisfied. 
By virtue of Lemma 7, one sees that the solution set for (GWVEP) can be represented by union of real set-valued $\xi(F)$ mappings. This means that the nonemptiness of $S_{\xi}^{P}(K, F)$ guarantees the existence of solution for (GWVEP). We next establish the existence theorem for $\xi$-weak efficient solution to the (GWVEP).

By the idea of linear scalarization technique, for any $\xi \in C^{* 0}$, we first introduce the set

$$
R_{1}^{\xi}:=\left\{d \in K_{\infty}: \sup _{z \in F(y, y+t d)} \xi(z) \leq 0, \forall y \in K, t>0\right\} .
$$

The following lemma shows that the condition of $\cup_{\xi \in C^{* 0}} R_{1}^{\xi}=\{0\}$ is weaker than $R_{1}=\{0\}$.

Lemma $8 \quad R_{1}=\{0\} \Rightarrow \cup_{\xi \in C^{* 0}} R_{1}^{\xi}=\{0\}$.

Proof Assume that $R_{1}=\{0\}$. Let $d_{0} \in \cup_{\xi \in C^{* 0}} R_{\xi}$. Then there exists $\xi_{0} \in C^{* 0}$ and $d_{0} \in K_{\infty}$ such that for every $y \in K$ and $t>0$

$$
\xi_{0}(z) \leq 0 \quad \text { for all } \quad z \in F\left(y, y+t d_{0}\right) .
$$

We claim that for any $z \in F\left(y, y+t d_{0}\right), z \notin$ int $C$. If not, there exists $z_{0} \in F\left(y, y+t d_{0}\right)$ such that $z \in \operatorname{int} C$ and so

$$
\xi_{0}\left(z_{0}\right)>0
$$

which leads to contradiction with (12). Hence, for every $y \in K$ and $t>0$

$$
F\left(y, y+t d_{0}\right) \cap(\operatorname{int} C)=\varnothing .
$$

By our hypothesis, $d_{0}=0$.

The following example shows that the inverse implication of Lemma 8 may not be true. The following example has been changed format.

Example 4 Let $X=\mathbb{R}, K=[0,+\infty), Y=\mathbb{R}^{2}, C=\mathbb{R}_{+}^{2}, e=(1,1) \in$ int $C$. Define $F: K \times K \rightarrow 2^{Y} \backslash\{\varnothing\}$ by

$$
F(x, y)= \begin{cases}\{0\} \times[0,1-|y-x|], & \text { if } 0 \leq|y-x| \leq 1, \\ {[|y-x|-1,0] \times\{0\},} & \text { if }|y-x|>1\end{cases}
$$

Then $K_{\infty}=[0,+\infty)$ and $C^{* 0}=\left\{\left(x_{1}, x_{2}\right) \in \mathbb{R}_{+}^{2}: x_{1}+x_{2}=1\right\}$.

We see that for any $y \in \mathbb{R}_{+}, d \in \mathbb{R}$ and $t>0$,

$$
F(y, y+t d) \subseteq \begin{cases}\{0\} \times[0,1], & \text { if } 0 \leq|t d| \leq 1, \\ {[0,+\infty) \times\{0\},} & \text { if }|t d|>1,\end{cases}
$$

which implies that $F(y, y+t d) \cap$ int $C=\varnothing$ for all $y \in \mathbb{R}_{+}, d \in \mathbb{R}$ and $t>0$. Hence, $R_{1}=[0,+\infty)$. But, for any $\xi \in C^{* 0}$, we have for any $y, d \in \mathbb{R}_{+}$and $t>0$

$$
\xi(z) \geq 0, \quad \text { for all } x \in F(y, y+t d),
$$


which implies that $d$ must be 0 , and so $R_{1}^{\xi}=\{0\}$ for all $\xi \in C^{* 0}$.

From the Corollary 1, we can obtain the following characterization corollary for $\xi$-efficient solution $S_{\xi}^{P}(K, F)$ and $S_{\xi}^{D}(K, F)$.

Corollary 2 Let $\xi \in C^{* 0}$ be any given. Let $K$ be a nonempty closed convex subset of $X$ and $F: K \times K \rightarrow 2^{Y} \backslash\{\varnothing\}$ be a set-valued mapping satisfying assumptions $\left(F_{0}\right),\left(F_{2}\right)-\left(F_{4}\right)$ and $(\mathrm{v})$ in Definition 3. Suppose that int $(\operatorname{barr}(K)) \neq \varnothing$. Then the following statements are equivalent:

(i) the solution set of $S_{\xi}^{P}(K, F)$ is nonempty and bounded;

(ii) the solution set of $S_{\xi}^{D}(K, F)$ is nonempty and bounded;

(iii) $R_{1}^{\xi}=\{0\}$;

(iv) there exists a bounded set $B \subset K$ such that for every $x \in K \backslash B$, there exists $y \in B$ such that $\xi(z)>0$ for some $z \in F(y, x)$.

Proof For any fixed $\xi \in C^{*} \backslash\{0\}$, we define $\xi(F): K \times K \rightarrow 2^{\mathbb{R}} \backslash\{\varnothing\}$ as in (9). It is not hard to check that $\xi(F)$ satisfies conditions $\left(F_{0}\right)-\left(F_{4}\right)$ in Corollary 1.

We now characterize the nonemptiness and boundedness of $S_{W}^{P}(K, F)$ in term of nonemptiness and boundedness of the solution set $S_{\xi}^{P}(K, F)$ for any $\xi \in C^{* 0}$.

Theorem 3 Let $X$ be a reflexive Banach space and $K$ be a closed convex subset of $X$ with $\operatorname{int}($ barrK $) \neq \varnothing$. Let $Y$ be a normed space and $C^{* 0}$ is a compact base of $C^{*}$. Suppose that $F: K \times K \rightarrow 2^{Y} \backslash\{\varnothing\}$ is a set-valued mapping satisfying assumptions $\left(F_{0}\right),\left(F_{2}\right)-\left(F_{4}\right)$ and (v) in Definition 3.

Then $S_{W}^{P}(K, F)$ is nonempty and bounded if and only iffor any $\xi \in C^{* 0}, S_{\xi}^{P}(K, F)$ is nonempty and bounded.

Proof Suppose that for any $\xi \in C^{* 0}, S_{\xi}^{P}(K, F)$ is nonempty and bounded. Then by Corollary $2, R_{1}^{\xi}=\{0\}$. We claim that $S_{W}^{P}(K, F)$ is nonempty and bounded. The nonemptiness of $S_{W}^{P}(K, F)$ is obvious, because of $S_{\xi}^{P}(K, F) \subset S_{W}^{P}(K, F)$. We only need to show that $S_{W}^{P}(K, F)$ is bounded. If not, there exists a sequence $x_{n} \in S_{W}^{P}(K, F)$ such that $\left\|x_{n}\right\| \rightarrow+\infty$. Since $x_{n} \in S_{W}^{P}(K, F)$, we then have

$$
F\left(x_{n}, y\right) \cap(-i n t C)=\varnothing, \text { for all } y \in K \text {. }
$$

Thus, for every $z_{n} \in F\left(x_{n}, y\right), z_{n} \notin-i n t C$. Then there exists $\xi_{n} \in C^{* 0}$ such that

$$
\xi_{n}\left(z_{n}\right) \geq 0, \text { for all } z \in F\left(x_{n}, y\right), \text { for all } y \in K .
$$

By the $\xi$-pseudomonotonicity of $F$, we have

$$
\xi_{n}\left(z_{n}^{\prime}\right) \leq 0, \quad \text { for all } z^{\prime} \in F\left(y, x_{n}\right), \text { for all } y \in K
$$

Since $C^{* 0}$ is compact, without loss of generality, we can assume that $\xi_{n} \rightarrow \xi_{0} \in C^{* 0}$. For any fixed $y \in K$ and $t>0$, without loss of generality, we may take a subsequence $\left\{x_{n_{k}}\right\}$ of $\left\{x_{n}\right\}$ such that 


$$
\frac{t}{\left\|x_{n_{k}}-y\right\|} \in(0,1) \quad \text { and } \quad w-\lim _{k \rightarrow+\infty} \frac{x_{n_{k}}-y}{\left\|x_{n_{k}}-y\right\|}=w-\lim _{k \rightarrow+\infty} \frac{x_{n_{k}}}{\left\|x_{n_{k}}\right\|}=d_{0} \in K_{\infty} .
$$

By Lemma $3, d_{0} \neq 0$. Upper $C$-convexity of $F$ implies

$$
\left(1-\frac{1}{\left\|x_{n_{k}}-y\right\|}\right) F(y, y)+\frac{t}{\left\|x_{n_{k}}-y\right\|} F\left(y, x_{n_{k}}\right) \subseteq F\left(y, y+\frac{t\left(x_{n_{k}}-y\right)}{\left\|x_{n_{k}}-y\right\|}\right)+C
$$

It follows from $F(y, y)=\{0\}$ and (14) that for any $\xi_{n}$,

$$
\begin{aligned}
\xi_{n}\left(F\left(y, y+\frac{t\left(x_{n_{k}}-y\right)}{\left\|x_{n_{k}}-y\right\|}\right)\right) & \leq\left(1-\frac{1}{\left\|x_{n_{k}}-y\right\|}\right) \xi_{n}(F(y, y))+\frac{t}{\left\|x_{n_{k}}-y\right\|} \xi_{n}\left(F\left(t, x_{n_{k}}\right)\right) \\
& \leq 0 .
\end{aligned}
$$

Since $F$ is weakly lower semicontinuous at second variable and $\xi_{n} \rightarrow \xi_{0}$, we have

$$
\xi_{0}\left(F\left(y, y+t d_{0}\right)\right) \leq 0 .
$$

This implies that $0 \neq d_{0} \in R_{1}^{\xi}$, which is a contradiction.

Conversely, we assume that $S_{W}^{P}(K, F)$ is nonempty and bounded. We claim that $S_{\xi}^{P}(K, F)$ is nonempty and bounded for all $\xi \in C^{* 0}$. We consider the set $A \subseteq C^{* 0}$ as follows.

$$
A:=\left\{\xi \in C^{* 0}: S_{\xi}^{P}(K, F) \text { is nonempty and bounded }\right\} .
$$

Clearly, $A$ is nonempty. Firstly, we claim that $A$ is open subset in $C^{* 0}$. If not, there exists $\xi_{0} \in A$ and a sequence $\xi_{n} \in C^{* 0}$ with $\xi_{n} \rightarrow \xi_{0}$ such that $\xi_{n} \notin A$. This implies that $R_{1}^{\xi_{n}} \neq\{0\}$. Then there exists $d_{n} \in R_{1}^{\xi_{n}}$ such that $\left\|d_{n}\right\|=1$. Since $C^{* 0}$ is compact and $\left\|d_{n}\right\|=1$, without loss of generality, we may assume that $d_{n} \rightarrow d_{0} \in K_{\infty} \backslash\{0\}$. Since $d_{n} \in R_{1}^{\xi_{n}}$, we have

$$
\xi_{n}\left(z^{\prime}\right) \leq 0 \text { for all } z^{\prime} \in F\left(y, y+t d_{n}\right) \text { for all } y \in K
$$

Since $F$ is weakly lower semicontinuous at second variable and $\xi_{n} \rightarrow \xi_{0}$, we have

$$
\xi_{0}\left(z^{\prime}\right) \leq 0 \text { for all } z^{\prime} \in F\left(y, y+t d_{0}\right) \text { for all } y \in K
$$

Thus $0 \neq d_{0} \in R \xi_{\xi_{0}}$. This implies that $S_{\xi_{0}}^{P}(K, F)$ is not nonempty and bounded, which leads to a contradiction with $\xi_{0} \in A$. Hence $A$ is an open subset of $C^{* 0}$.

Finally, we claim that $A$ is a closed subset of $C^{* 0}$. Let $\xi_{n} \in A$ with $\xi_{n} \rightarrow \xi_{0}$. In view of $\xi_{n} \in A$, we have $S_{\xi_{n}}(K, F)$ is nonempty and bounded. Let $x_{n} \in S_{\xi_{n}}^{P}(K, F)$. Whereas $S_{\xi_{n}}^{P}(K, F) \subset S_{W}^{P}(K, F)$ and $S_{W}^{P}(K, F)$ is bounded, $\left\{x_{n}\right\}$ is also. We may assume that $x_{n} \rightarrow x_{0} \in K$. Since $x_{n} \in S_{\xi_{n}}(K, F)$, then we have

$$
\xi_{n}(z) \geq 0 \text { for all } z \in F\left(x_{n}, y\right) \text { for all } y \in K .
$$

By $\xi$-pseudomonotonicity of $F$, we get

$$
\xi_{n}\left(z^{\prime}\right) \leq 0, \quad \text { for all } z^{\prime} \in F\left(y, x_{n}\right), \text { for all } y \in K .
$$

Since $F$ is weakly lower semicontinuous at the second variable, letting $n \rightarrow \infty$

$$
\xi_{0}\left(z^{\prime}\right) \leq 0, \quad \text { for all } z^{\prime} \in F\left(y, x_{0}\right), \text { for all } y \in K
$$


Hence, $x_{0} \in S_{\xi_{0}}^{D}(K, F)$. Thanks to Corollary 2, we get that $x_{0} \in S_{\xi_{0}}^{P}(K, F)$. The boundedness of $S_{W}^{P}(K, F)$ implies $S_{\xi_{0}}^{P}(K, F)$ is also. This means that $\xi_{0} \in A$ and so $A$ is closed. Since the base $C^{* 0}$ of $C^{*}$ is connected, we have $A$ must be $C^{* 0}$.

Theorem 4 Let $X$ be a reflexive Banach space and $K$ be a closed convex subset of $X$ with $\operatorname{int}($ barrK $) \neq \varnothing$. Let $Y$ be a normed space and $C^{* 0}$ is a compact base of $C^{*}$. Suppose that $F: K \times K \rightarrow 2^{Y} \backslash\{\varnothing\}$ is a set-valued mapping satisfying assumptions $\left(F_{0}\right),\left(F_{2}\right)-\left(F_{4}\right)$ and (v) in Definition 3. Then the following statements are equivalent.

(i) $S_{W}^{P}(K, F)$ is nonempty and bounded;

(ii) For every $\xi \in C^{* 0}, S_{\xi}^{P}(K, F)$ is nonempty and bounded;

(iii) $\cup_{\xi \in C^{* 0}} R_{1}^{\xi}=\{0\}$.

Remark 3 Theorem 4 generalize Theorem 2, in the following three cases:

(i) Condition $\left(F_{1}\right)$ is relaxed to the condition $\left(F_{1}^{\xi}\right)$.

(ii) Recession cone $R_{1}=\{0\}$ is relaxed to the condition $\cup_{\xi \in C^{* 0}} R_{1}^{\xi}=\{0\}$.

(iii) Condition $\left(F_{5}\right)$ is omitted.

The following example show that Theorem 4 is applicable.

Example 5 Let $X=\mathbb{R}, K=[0,+\infty), Y=\mathbb{R}^{2}, C=\mathbb{R}_{+}^{2}, e=(1,1) \in$ int $C$. A set-valued $\operatorname{map} F: K \times K \rightarrow 2^{\mathbb{R}^{2}} \backslash\{\varnothing\}$ is defined by

$$
F(x, y)=\{(y-x)\} \times\left[(y-x),\left(e^{(y-x)}-1\right)+(y-x)\right]
$$

Then, clearly $\left(F_{0}\right),\left(F_{2}\right)-\left(F_{4}\right)$ and $(\mathrm{v})$ in Definition 3 are satisfied. For any $\xi \in C^{* 0}$, we consider

$$
\begin{aligned}
R_{1}^{\xi} & =\left\{d \in K_{\infty}: \sup _{z \in(F(y, y+t d))} \xi(z) \leq 0, \forall y \in K, t>0\right\} \\
& =\left\{d \in[0,+\infty): \xi(z) \leq 0, \forall z \in\{t d\} \times\left[t d,\left(e^{t d}-1\right)+t d\right] \text { and } \forall y \in K, t>0\right\}=\{0\} .
\end{aligned}
$$

It follows from Theorem 4 that, $S_{W}^{P}(K, F)$ is nonempty and bounded.

\section{Stability analysis}

In this section, we shall establish the stability theorem of solution set for (GWVEP) when the mapping $F$ and the domain set $K$ are perturbed by different parameters.

We first recall some important notions . Let $\left(\Lambda, d_{\Lambda}\right)$ and $\left(M, d_{M}\right)$ be two metric spaces. Let $K(\lambda)$ be perturbed by a parameter $\lambda$, which varies over $\left(\Lambda, d_{\Lambda}\right)$, that is, $K: \Lambda \rightarrow 2^{X}$ is a set-valued mapping with nonempty, closed, and convex values. Let $F$ be perturbed by a parameter $\mu$, which varies over $\left(M, d_{M}\right)$, that is, $F: K \times K \times M \rightarrow 2^{Y} \backslash\{\varnothing\}$ is a parametric set-valued mapping.

Consider the parametric generalized weak vector equilibrium problems, denoted by (PGWVEP), which consists in finding $\bar{x} \in K(\lambda)$ such that

$$
F(\bar{x}, y, \mu) \cap(-\operatorname{int} C) \neq \varnothing \quad \forall y \in K(\lambda) .
$$

(PGWVEP) 
Denote by $S_{W}^{P}(\lambda, \mu)$ the set of all weak efficient solution to the (PGWVEP).

Let $\left\{A_{n}\right\}$ be a sequence of nonempty subset of $X$. We define

$$
\limsup _{n \rightarrow+\infty} A_{n}:=\left\{x \in X: \exists\left\{n_{k}\right\}, x_{n_{k}} \in A_{n_{k}} \text { such that } x_{n_{k}} \rightarrow x\right\} .
$$

We say that the sequence $\left\{A_{n}\right\}$ is of upper convergence in the sense of Painlevie-Kuratowski (P.K. convergence) Durea (2007) to $A$ if $\lim \sup _{n \rightarrow+\infty} A_{n} \subseteq A$.

The following theorem shows that under suitable situation, there exists a neighborhood $N\left(\lambda_{0}\right) \times N\left(\mu_{0}\right)$ of $\left(\lambda_{0}, \mu_{0}\right)$ such that $S_{W}^{P}(\lambda, \mu)$ P.K. convergence to $S_{W}^{P}\left(\lambda_{0}, \mu_{0}\right)$ in the neighborhood $N\left(\lambda_{0}\right) \times N\left(\mu_{0}\right)$.

Theorem 5 Let $X$ be a real reflexive Banach space and $K$ be a closed convex subset of $X$ with int $($ barrK $) \neq \varnothing$. Let $Y$ be a normed space and $C^{* 0}$ is a compact base of $C^{*}$. Suppose that $F$ satisfies the following conditions:

(I) $K(\cdot)$ is continuous on $\Lambda$ and $\operatorname{int}\left(\operatorname{barr} K\left(\lambda_{0}\right)\right) \neq \varnothing$, for all $\lambda \in \Lambda$ and has nonempty closed convex valued.

(II) For any $\lambda \in \Lambda$ and $x \in K(\lambda), F(x, x, \mu)=\{0\}$.

(III) For any $\lambda \in \Lambda$ and $\mu \in M, F(\cdot, \cdot, \mu)$ is $\xi$-pseudomonotone on $K(\lambda)$ w.r.t. $C^{* 0}$.

(IV) For any $\mu \in M$ and $x \in K(\mu), F(x, \cdot, \mu)$ is $C$-convex.

(V) For any $\lambda \in \Lambda$ and $\mu \in M, F(\cdot, \cdot, \cdot)$ is continuous on $K(\lambda) \times K(\lambda) \times M$.

If $S_{W}^{P}\left(\lambda_{0}, \mu_{0}\right)$ is nonempty and bounded, then the following statements hold.

(i) there exists a neighborhood $N\left(\lambda_{0}\right) \times N\left(\mu_{0}\right)$ such that $S_{W}^{P}(\lambda, \mu)$ has a nonempty and bounded for all $(\lambda, \mu) \in N\left(\lambda_{0}\right) \times N\left(\mu_{0}\right)$.

(ii) $\lim \sup _{(\lambda, \mu) \rightarrow\left(\lambda_{0}, \mu_{0}\right)} S_{W}^{P}(\lambda, \mu) \subseteq S_{W}^{P}\left(\lambda_{0}, \mu_{0}\right)$.

Proof (i) We claim that there exists a neighborhood $N\left(\lambda_{0}\right) \times N\left(\mu_{0}\right)$ of $\left(\lambda_{0}, \mu_{0}\right)$ such that for any $(\lambda, \mu) \in N\left(\lambda_{0}\right) \times N\left(\mu_{0}\right)$ and $\xi \in C^{* 0}$

$$
R_{1}^{\xi}(\lambda, \mu):=\left\{d \in K(\lambda)_{\infty}: \sup _{z \in(F(y, y+t d, \mu))} \xi(z) \leq 0, \forall y \in K, t>0\right\}=\{0\} .
$$

If not, there exists $\left(\lambda_{n}, \mu_{n}\right) \in \Lambda \times M$ with $\left(\lambda_{n}, \mu_{n}\right) \rightarrow\left(\lambda_{0}, \mu_{0}\right)$ and $\xi^{\prime} \in C^{* 0}$ such that $R_{1}^{\xi^{\prime}}\left(\lambda_{n}, \mu_{n}\right) \neq\{0\}$.

Since $K$ is lower semicontinuous at $\lambda_{0}$, for any $y \in K\left(\lambda_{0}\right)$, we have $y_{n} \in K\left(\lambda_{n}\right)$ such that $y_{n} \rightarrow y$. Together with $\mu_{n} \rightarrow \mu_{0}$, we have $\left(y_{n}, \mu_{n}\right) \rightarrow\left(y, \mu_{0}\right)$. Thus, we can select a sequence $\left\{d_{n}\right\}$ such that

$$
d_{n} \in K\left(\lambda_{n}\right)_{\infty} \text { and } \sup _{z \in F\left(y_{n}, y_{n}+t d_{n}, \mu_{n}\right)} \xi^{\prime}(z) \leq 0, \quad \forall y \in K\left(\lambda_{n}\right), t>0 .
$$

with $\left\|d_{n}\right\|=1$ for all $n=1,2, \ldots$. Since $\left\{d_{n}\right\}$ is a bounded sequence in a reflexive Banach space $X$ we can assume that $d_{n} \rightarrow d_{0}$. It follows from Lemma 4 that $d_{0} \neq 0$. We claim that $d_{0} \in K\left(\lambda_{0}\right)_{\infty}$. Since $K$ is upper semicontinuous at $\lambda_{0}$ and $d_{n} \in K\left(\lambda_{n}\right)_{\infty}$, by Lemma 5 , 
we have that $d_{n} \in K\left(\lambda_{0}\right)_{\infty}$, for all sufficiently large $n$. By the closure of $K\left(\lambda_{0}\right)_{\infty}$, we have $d_{0} \in K\left(\lambda_{0}\right)_{\infty}$. Notice that the continuity assumption of $F$, taking the limit in (15), we have

$$
\sup _{z \in F\left(y, y+t d_{0}, \mu_{0}\right)} \xi^{\prime}(z) \leq 0
$$

which implies that $0 \neq d_{0} \in R_{1}^{\xi^{\prime}}\left(\lambda_{0}, \mu_{0}\right)$. This is a contradiction with $S_{W}^{P}\left(\lambda_{0}, \mu_{0}\right) \neq \varnothing$, so we have the claim.

(ii) We want to show that for any $(\lambda, \mu) \rightarrow\left(\lambda_{0}, \mu_{0}\right)$,

$$
\limsup _{(\lambda, \mu) \rightarrow\left(\lambda_{0}, \mu_{0}\right)} S_{W}^{P}(\lambda, \mu) \subseteq S_{W}^{P}\left(\lambda_{0}, \mu_{0}\right) .
$$

Let $\bar{x} \in \lim \sup _{(\lambda, \mu) \rightarrow\left(\lambda_{0}, \mu_{0}\right)} S_{W}^{P}(\lambda, \mu)$. Then there exits a sequence $x_{n_{k}} \in S_{W}^{P}\left(\lambda_{n_{k}}, \mu_{n_{k}}\right)$ such that $x_{n_{k}} \rightarrow \bar{x}$ as $k \rightarrow \infty$. Since $K$ is upper semicontinuous at $\lambda_{0}$, for sufficiently large $n$ we get that

$$
K\left(\lambda_{n}\right) \subseteq K\left(\lambda_{0}\right)+\frac{1}{n} B
$$

where $B$ is a closed unit ball. By virtue of $x_{n_{k}} \in K\left(\lambda_{n_{k}}\right)$, we get that

$$
d\left(x_{n_{k}}, K\left(\lambda_{0}\right)\right) \leq \frac{1}{n_{k}} \rightarrow 0 .
$$

It follows from $K\left(\lambda_{0}\right)$ is closed and $x_{n_{k}} \rightarrow \bar{x}$ that $\bar{x} \in K\left(\lambda_{0}\right)$.

Since $K$ is lower semicontinuous at $\lambda_{0}$, for any $y \in K\left(\lambda_{0}\right)$ there exists $y_{n_{k}} \in K\left(\lambda_{n_{k}}\right)$ with $y_{n_{k}} \rightarrow y$. By our hypothesis, we get

$$
F\left(x_{n_{k}}, y_{n_{k}}, \mu_{n_{k}}\right) \cap(-i n t C)=\varnothing .
$$

Continuity of $F$ implies

$$
F\left(\bar{x}, y, \mu_{0}\right) \cap(-\operatorname{int} C)=\varnothing .
$$

Since the latest inequality holds for all $y \in K\left(\lambda_{0}\right)$. Hence, $\bar{x} \in S_{W}^{P}\left(\lambda_{0}, \mu_{0}\right)$.

\section{Conclusions}

In this paper, some characterizations of nonemptiness and boundedness of solution sets for generalized weak vector equilibrium problems are established in a reflexive Banach space. By using the linear scalarization method, we give a sufficient and necessary condition for the nonemptiness and boundedness of $S_{W}^{P}(K, F)$ in term of nonemptiness and boundedness of the solution set $S_{\xi}^{P}(K, F)$ for any $\xi \in C^{* 0}$. As application, we discuss the stability result for the solution set to (PGWVEP) in the sense of Painlevé-Kuratowski upper convergence of set. 


\section{Author details}

1 Program in Mathematics, Faculty of Education, Pibulsongkram Rajabhat University, Phitsanulok 65000, Thailand.

2 Department of Mathematics, Faculty of Science, Naresuan University, Phitsanulok 65000, Thailand.

\section{Acknowledgements}

The authors would like to thank the referees for reading this paper carefully, providing valuable suggestions and comments in the original version of this paper. The first author was supported by Thailand Research Fund (TRG5880058).

\section{Competing interests}

Both authors declare that they have no competing interests.

Received: 30 March 2016 Accepted: 4 August 2016

Published online: 12 August 2016

\section{References}

Adly S, Ernst E, Théra M (2004) Well-positioned closed convex sets and well-positioned closed convex functions. J Glob Optim 29:337-351

Ansari QH, Konnov IV, Yao JC (2001) On generalized vector equilibrium problems. Nonlinear Anal 47:543-554

Ansari QH, Konnov IV, Yao JC (2001) Existence of a solution and variational principles for vector equilibrium problems. J Optim Theory Appl 110:481-492

Ansari QH, Siddiqi AH, Wu SY (2001) Existence and duality of generalized vector equilibrium problems. J Math Anal Appl 259:115-126

Ansari QH, Konnov IV, Yao JC (2002) Characterizations of solutions for vector equilibrium problems. J Optim Theory Appl 113:435-447

Ansari QH, Flores-Bazán F (2006) Recession methods for generalized vector equilibrium problems. J Math Anal Appl $321: 132-146$

Aubin JP, Ekeland I (1984) Applied nonlinear analysis. Wiley, New York

Chen B, Gong XH, Yuan SM (2008) Connectedness and compactness of weak efficient solutions for set-valued vector equilibrium problems. J Inequal Appl. doi:10.1155/2008/581849

Chen CR, Li SJ, Teo KL (2009) Solution semicontinuity of parametric generalized vector equilibrium problems. J Glob Optim 45:309-318

Durea M (2007) On the existence and stability of approximate solutions of perturbed vector equilibrium problems. J Math Anal Appl 333:1165-1179

Fan K (1984) Some properties of convex sets related to fixed point theorems. Math Ann 266:519-537

Fang YP, Huang NJ (2007) Equivalence of equilibrium problems and least element problems. J Optim Theory Appl 132:411-422

Fan JH, Zhong RY (2008) Stability analysis for variational inequality in reflexive Banach spaces. Nonlinear Anal 8:2566-2574

Ferro F (1989) A minimax theorem for vector-valued function. J Optim Theory Appl 60:19-31

Flores-Bazán F (2000) Existence theorems for generalized noncoercive equilibrium problems: the quasiconvex case. SIAM J Optim 11:675-690

Flores-Bazán F, Flores-Bazán F (2003) Vector equilibrium problems under asymptotic analysis. J Glob Optim 26:141-166

Gong XH (2008) Continuity of the solution set to parametric weak vector equilibrium problems. J Optim Theory Appl 139:35-46

Huang XX, Fang YP, Yang XQ (2014) Characterizing the nonemptiness and compactness of the solution set of a vector variational inequality by scalarization. J Optim Theory Appl 162:548-558

Li SJ, Li XB (2011) Hölder continuity of solutions to parametric weak generalized Ky Fan inequality. J Optim Theory Appl 149:540-553

Sadeqi I, Alizadeh CG (2011) Existence solutions of generalized vector equilibrium problems in reflexive Banach spaces. Nonlinear Anal 74:2226-2234

Xu YD, Li SJ (2013) On the lower semicontinuity of the solution mappings to a parametric generalized strong vector equilibrium problem. Positivity 17:341-353

Zhong RY, Huang NJ, Cho YJ (2011) Boundedness and nonemptiness of solution sets for set-valued vector equilibrium problems with application. J Inequal Appl. doi:10.1155/2011/936428 